\section{OP80 GUIDANCE FOR ADAPTING EVIDENCE-INFORMED POPULATION HEALTH INTERVENTIONS FOR NEW CONTEXTS: THE ADAPT STUDY}

${ }^{1} \mathrm{G}$ Moore*, 'L Copeland, ${ }^{2} \mathrm{~A}$ Movsisyan, ${ }^{3} \mathrm{M}$ Campbell, ${ }^{3} \mathrm{P}$ Craig, ${ }^{1} \mathrm{R}$ Evans. ${ }^{1} D E C I P H e r$, School of Social Sciences, Cardiff University, Cardiff, UK; ${ }^{2}$ Institute for Medical Information Processing, Biometry and Epidemiology, Ludwig Maximilian University of Munich, Munich, Germany; ${ }^{3}$ MRC/CSO Social and Public Health Sciences Unit, University of Glasgow, Glasgow, UK

\subsection{6/jech-2020-SSMabstracts.79}

Background Adaptation of evidence-informed population health interventions for new contexts may be more efficient than de-novo intervention development, where well developed and tested interventions exist. There is however currently no consensus-based guidance for decision-making on adapting interventions for implementation and/or deciding on the need for re-evaluation in new contexts. We are developing guidance, with funding from MRC-NIHR. This presentation will describe the underpinning research used to develop this guidance, and draft content of the guidance, which will be completed by the end of 2020 .

Methods We used a phased approach to develop guidance. This began with a systematic review of existing guidance, including papers published between January 2000 and October 2018 identified through searches of 7 databases. This was followed by a scoping review focused on a purposive sample of cases of intervention adaptation identified through this search. Semi-structured qualitative interviews with researchers $(\mathrm{N}=23)$, practitioners $(\mathrm{N}=3)$, journal editors $(\mathrm{N}=5)$ and funders $(\mathrm{N}=6)$, were conducted, and analysed using framework analysis. Finally, a three round modified Delphi consensus process was undertaken $(\mathrm{N}=66)$.

Results The systematic review identified and synthesised 35 sources of guidance to develop a draft framework, which was refined in subsequent phases. Reviews and qualitative interviews identified several ongoing debates in adapting interventions for new contexts, and areas of consensus. Informed by the review, we defined adaptation as intentional modification(s) of an evidence-informed intervention, in order to achieve better fit between an intervention and a new context'. Consensus processes suggested this definition was useful, but emphasised the importance of including guidance for both pro-active and re-active adaptation. Different conceptualisations of fidelity were identified within the reviews and interviews which shaped approaches to adapting interventions. Further, while we initially defined 'evidenceinformed' interventions as those with prior evidence of effects, qualitative interviews and DELPHI processes suggested a wider framing of 'evidence-informed', with interventions often adapted from elsewhere based on evidence of feasibility, but with no prior robust evaluation of effects. Draft recommendations for adapting interventions for new contexts include considerations in relation to what interventions to select, when and how to adapt these, the level of re-evaluation required in the new context, and reporting of adaptation processes and outcomes.

Discussion Producers and users of population health evidence face significant uncertainties over whether and how to adapt and re-evaluate interventions in new contexts. This guidance should improve the commissioning, conduct and reporting of studies involving intervention adaptation for new contexts.

\section{OP81 \\ DEVELOPMENT OF BEHAVIOUR CHANGE INTERVENTION TO PROMOTE PHYSICAL ACTIVITY FOR CANCER SURVIVORS: A MIXED METHOD APPROACH USING MRC FRAMEWORK}

${ }^{1} S$ Nafees*, ${ }^{2} \mathrm{~J}$ Garzoni, ${ }^{3} \mathrm{~N}$ Din, ${ }^{3} \mathrm{~J}$ Hiscock, ${ }^{3} \mathrm{M}$ Hendry, ${ }^{4} \mathrm{RT}$ Edwards, ${ }^{5} \mathrm{~N}$ Williams. ${ }^{1}$ CFMHAS, Bangor University, Wrexham, UK; ${ }^{2}$ Vascular Network, Betsi Cadwaladr University Health Board, Glan Clwyd, UK; ${ }^{3}$ NWCPCR, Bangor University, Wrexham, UK; ${ }^{4}$ CHEME, Bangor University, Bangor, UK; ${ }^{5}$ Department of Health Services Research, Liverpool University, Liverpool, UK

\subsection{6/jech-2020-SSMabstracts. 80}

Background Although beneficial effects of physical activity in adult cancer survivors are well-established, patients are often not active enough to reap such benefits. The aim of this project was to develop a theory-based physical activity behaviour change intervention to promote physical activity in cancer survivors.

Methods Development stage of the MRC framework was completed conducting systematic review with meta-analysis, focus groups of adult survivors of any type of cancer and an online survey of primary, secondary and allied healthcare professionals in oncology. A separate literature review was conducted to identify the relevant theory to underpin the proposed physical activity promotional intervention.

Results Education materials in various formats are effective to increase physical activity in cancer survivors. Reminders based on behaviour change theories complements readiness to initiate or increase exercise. Focus group participants in general revealed receiving inconsistent physical activity advice, and preferred this to be tailored and face-to-face as part of their standard care, with a component of reminders at the end of their active cancer treatment. The survey findings reiterated focus group participants' experiences highlighting such inconsistency, partly because of health professionals' lack of knowledge or confidence to offer optimum physical activity advice.

Findings from the above three phases were integrated into a weekly text message based intervention that can easily be integrated into existing health services with minimal structural or financial implications.

Conclusion Physical inactivity in cancer survivors needs addressing to improve their quality of life. Behaviour change interventions need to be theory-driven and low-maintenance. The proposed intervention to promote physical activity in cancer survivors comprising of 12 weekly text messages may be beneficial when integrated into existing health services.

\section{OP82 HEALTH, SOCIAL CARE AND TECHNOLOGICAL INTERVENTIONS TO IMPROVE FUNCTIONAL ABILITY AMONG OLDER ADULTS: A CAMPBELL EVIDENCE AND GAP MAP}

1) Thompson Coon, ${ }^{2} \mathrm{~V}$ Welch, ${ }^{3} \mathrm{TE}$ Howe, ${ }^{4} \mathrm{~S}$ Marcus, ${ }^{5} \mathrm{CM}$ Mathew ${ }^{*},{ }^{6} \mathrm{R}$ Sadana. ${ }^{1} \mathrm{NIHR}$ ARC South West Peninsula, University of Exeter Medical School, Exeter, UK; ${ }^{2}$ Methods Centre, Bruyere Research Insitute, Ottawa, Canada; ${ }^{3}$ City of Glasgow College, Glasgow, UK; ${ }^{4}$ Radcliffe Department of Medicine, University of Oxford, Oxford, UK; ${ }^{5}$ Bruyere Research Institute, Ottawa, Canada; ${ }^{6}$ World Health Organization, Geneva, Switzerland

\subsection{6/jech-2020-SSMabstracts.81}

Background By 2030, adults over 60 years are expected to be higher than the number of children under 10 years globally. Currently, over two thirds of people over 65 years of age are living with multi-morbidities. With increasing disparities in health care and determinants of health, there are major health 